\title{
Gobernanza lateral de redes: Legitimidad y delegación relacional de la autoridad decisoria
}

\author{
Lateral Network Governance: Legitimacy and the \\ Relational Delegation of Decision-Making Authority
}

Johannes Glückler ${ }^{1}$

\begin{abstract}
RESUMEN
Este artículo va más allá del reconocimiento de las redes como un modo de gobernanza para elaborar sobre las formas reales de gobernanza que transmiten una coordinación legítima y aceptable. Avanza el concepto de gobernanza lateral de redes en el contexto empírico de las redes organizadas, en el que las empresas ponen en común sus recursos y unen sus intereses en la búsqueda de objetivos comunes. Para resolver el rompecabezas de tener iguales independientes que se comprometan a coordinar sus acciones, el documento pretende superar el dualismo tradicional entre los mecanismos formales e informales de gobernanza. En su lugar, concibe la gobernanza lateral de la red como una estructura para la delegación legítima de la toma de decisiones. Desarrolla un enfoque analítico de redes sociales para evaluar la distribución relacional de la legitimidad. El análisis empírico de dos estudios de caso de organizaciones de redes entre empresas ilustra el grado en que la distribución real de la legitimidad difiere de la autoridad formal de gobernanza. La gobernanza lateral de redes tiene consecuencias prácticas para los gerentes de redes.
\end{abstract}

Palabras clave: Gobernanza de redes, legitimidad, redes interorganizacionales, análisis de redes sociales, gobernanza regional

\begin{abstract}
This article goes beyond the acknowledgement of networks as a governance mode to elaborate on the actual forms of governance that convey legitimate and acceptable coordination. It advances the concept of lateral network governance in the empirical context of organized networks, in which firms pool resources and join their interests in the pursuit of common goals. To solve the puzzle of having independent equals commit themselves to coordinating their actions, the paper aims to overcome the traditional dualism between formal and informal mechanisms of governance. Instead it conceives lateral network governance as a structure for the legitimate delegation of decision-making. It develops a social network analytic approach to assessing the relational distribution of legitimacy. The empirical analysis of two case studies of inter-firm network organizations illustrates the degree to which the actual legitimacy distribution diverges from formal governance authority. Lateral network governance has practical implications for inter-organizational networks and network managers.
\end{abstract}

Keywords: Network governance, legitimacy, inter-organizational networks, social network analysis, regional governance. 


\section{Introducción}

Cuando las empresas se organizan en redes interorganizacionales, necesitan acordar objetivos comunes y una forma viable de trabajar entre ellas. ¿Cómo se deben diseñar y gobernar las redes para ayudar a los socios a alcanzar sus objetivos comunes de manera eficiente? Esta pregunta es particularmente vibrante para las organizaciones que se esfuerzan por superar los dilemas de la acción colectiva a nivel local, así como a través de escalas geográficas, para establecer prácticas legítimas de cooperación y cumplimiento en diversos contextos, como el ambiental (Liverman, 2004; Bulkeley, 2005; Scott, 2015) y la gobernanza regional (Macleod \& Goodwin, 1999; Feiock, 2007; Montero \& Chapple, 2019), la gobernanza de los bienes comunes locales (Hardin, 1968; Ostrom, 1999) y en los nuevos mecanismos organizativos - a menudo locales - de las llamadas economías diversas (Gibson-Graham, 2008) y las prácticas económicas alternativas (Seyfang, 2006; Sánchez Hernández, 2019). Es aquí donde la investigación en geografía humana, estudios de organización, ciencias políticas, sociales y ambientales, así como en la ciencia y análisis de redes, se cruzan para concebir soluciones para una gobernanza exitosa entre diversos grupos de actores. Si bien la investigación ha establecido una comprensión de las redes como un modo alternativo de gobernanza frente a los mercados y las jerarquías (Williamson, 1985; Powell, 1991), todavía sabemos poco acerca de cómo deben ser gobernadas realmente las organizaciones de redes. Para ser más precisos, lo que se necesita es comprender qué formas de gobernanza de la red son adecuadas en qué contextos de cooperación inter-organizativa: “Así pues, nos queda la comprensión de por qué las redes pueden ser un modo superior de gobierno, pero no de cómo se gobiernan a sí mismas" (Provan et al., 2007, 504).

Es prematuro llamar al campo de investigación sobre la gobernanza de la red un marco de investigación coherente (Glückler et al., 2012; Maggetti \& Gilardi, 2014; Jung et al., 2015; Ansell \& Torfing, 2016; Keast, 2016; Prota, 2016), aún más, porque el término gobernanza se ha utilizado de manera bastante amplia en las ciencias sociales, como en las ciencias políticas (Crouch, 2005; Rhodes, 2007; Ansell \& Gash, 2008), la economía institucional (Williamson, 2005), la sociología (Powell, 1991; Podolny \& Page, 1998; Rowley et al., 2000), y la geografía humana (Macleod \& Goodwin, 1999; Allen \& Cochrane, 2007; Charron et al., 2014). La escasez de conceptos y de conocimientos empíricos sobre la gobernanza en red se debe principalmente a las aspiraciones igualitarias de los socios que cooperan en redes laterales. A diferencia de las denominadas redes estratégicas (Sydow et al., 2016) o de las redes de valor añadido estructuradas verticalmente, como las cadenas de valor mundiales (Gereffi et al., 2005; Crang et al., 2013; Glückler \& Panitz, 2016; Grabs \& Ponte, 2019) o las redes de producción mundiales (Coe et al., 2008; Levy, 2008; Yeung \& Coe, 2015), en las que las funciones de gobernanza a menudo son asumidas por una empresa líder, las redes laterales están compuestas por miembros que se consideran autónomos e iguales a los demás. Por lo tanto, los miembros de las redes laterales a menudo desconfían del control externo: "Hay poco trabajo empíricamente fundamentado que investigue cómo pueden operar las organizaciones sin jefes y seguidores permanentes, en las que todos los miembros tienen formalmente la misma voz en la conducción de las operaciones o en el ejercicio del control" (Lazega, 2001, p. 1).

En este artículo, me gustaría avanzar el concepto de gobernanza en redes laterales en un contexto empírico específico de redes interorganizacionales que ha recibido sólo una atención limitada en la investigación sobre gobernanza: la red organizada. Una red organizada es una asociación voluntaria y deliberada de miembros, que dirige la cooperación multilateral entre un número limi- 
tado de organizaciones jurídica y económicamente independientes hacia un objetivo económico común (Glückler, 2012). Las expectativas de ejercer de manera autónoma el control en asociación con los demás son inconsistentes con las rígidas jerarquías de toma de decisiones o imposibles de cumplir, ya que una gobernanza débil no puede garantizar el cumplimiento. Este artículo se centra en la paradoja de tener iguales que se comprometen a coordinar sus acciones. Para resolver este problema fundamental, propongo analizar la gobernanza en red como una estructura para la delegación legítima de la toma de decisiones. La gobernanza lateral que surge de dicha transferencia tiene en cuenta tanto la contextualidad como la maleabilidad de las redes y permite dar a los mecanismos de gobernanza formales e informales la misma consideración y al mismo tiempo. La estructura para la transferencia legítima de la toma de decisiones, como ilustrará el análisis empírico, sienta las bases para una gobernanza eficaz y legítima de las redes organizadas.

La primera sección propone un marco de cuatro elementos -contexto, objeto, mecanismo y agencia de gobernanza- que en conjunto compensan la variación empírica en las formas de gobernanza. Partiendo de la noción de la distribución relacional de legitimidad, la segunda sección desarrolla el concepto de gobernanza de redes laterales. Ayuda a identificar a los agentes legítimos de la gobernanza al captar la delegación de autoridad discrecional entre iguales. La tercera sección desarrolla un método para evaluar empíricamente la transferencia legítima de la autoridad de toma de decisiones a partir de conceptos del análisis de redes sociales. En la cuarta sección, dos redes de pequeñas y medianas empresas (PYMES) -una en el ámbito de la consultoría de gestión y otra en el de la tecnología odontológica- muestran empíricamente hasta qué punto la distribución relacional de la legitimidad realmente observada puede diferir de la estructura de gobernanza formal. Un concepto de gobernanza en redes laterales y el análisis de las estructuras relacionales de legitimidad sugieren una comprensión más holística de la gobernanza de redes que integra posiciones formales, órganos y contratos con relaciones sociales informales de autoridad y legitimidad.

\section{El reto de la gobernanza}

Una de las razones de la diversidad de la investigación sobre la gobernanza en red es la gran variación en los contextos y fenómenos empíricos que se estudian actualmente. Una teoría más general de la gobernanza en red, por lo tanto, requiere una terminología consistente y necesita capturar el contexto empírico particular que distingue a las redes de las organizaciones jerárquicas como las corporaciones o las burocracias. Las formas de gobernanza de la red varían en función de al menos cuatro elementos clave que juntos constituyen el complejo de la gobernanza de la red: Estos son: i) el contexto de la gobernanza, es decir, las condiciones que enmarcan la colaboración entre actores; ii) el objeto de la gobernanza, es decir, el interés común y los objetivos de la colaboración; iii) los mecanismos de gobernanza, es decir, los contratos, el poder, la confianza, etc.; y iv) el organismo de gobernanza, es decir, los agentes y órganos a los que se ha concedido la autorización o legitimidad para ejercer la gobernanza.

\section{El contexto de la gobernanza: Cooperación en red}

Cuando se trata de redes organizadas como se ha definido anteriormente, algunos de los factores contextuales más importantes son las expectativas de los socios legalmente independientes 
e iguales en un grupo voluntario (Staber, 2000). Esto es tanto más pertinente en el contexto de las redes horizontales, en las que las empresas operan en fases iguales o similares de la cadena de valor. Por un lado, las redes horizontales representan espacios para la competencia potencial o real entre los miembros en sus respectivos mercados de factores y productos básicos. Por otro lado, las firmas miembro -a diferencia de las que están en redes estratégicas o redes verticales de valor añadido - no dependen unas de otras para sus recursos. La autonomía jurídica, la competencia potencial y la independencia mutua con respecto a los recursos de la otra parte constituyen un contexto muy distinto. Esta constelación de factores plantea un gran desafío para la gobernanza en red, ya que excluye los principios jerárquicos como mecanismos de control (Lazega, 2000; Lazega \& Krackhardt, 2000). La base voluntaria y autónoma de la acción dificulta la emisión de directivas jerárquicas, que los miembros pueden aceptar, pero que de ninguna manera deben aceptar. Lo mismo ocurre con las sanciones. Incluso si se acuerda contractualmente que el comportamiento indeseable en la red puede ser sancionado, la aplicación de estas sanciones formales puede poner en peligro la red en la medida en que los miembros puedan abandonarla, una salida que podría eventualmente desestabilizarla (Lazega, 2000). La afiliación voluntaria se basa en el beneficio que una persona espera de ella. Los miembros de la red vinculan la ampliación de su membresía a su evaluación de si los beneficios que otorga pueden compensar sus costos. La voluntad de opinar es, por lo tanto, relativamente alta, y el objetivo de la red debe ser objeto de un debate constante en el que los miembros construyan un consenso. Ring \& Van De Ven (1994) sostienen que los miembros de la red tienen que desarrollar un concepto común, no individual, de su motivación, sus inversiones viables y las probables incertidumbres, un proceso típico de la etapa de negociación de los modelos de madurez de la evolución de la red. Aunque los estudios empíricos no corroboran necesariamente la validez de ese modelo de madurez, la negociación de un consenso sobre los objetivos y la gobernanza tiene una importante influencia en la comprensión de la red.

\section{El objeto de gobernanza}

El segundo elemento de la gobernanza en red es el objeto de ser gobernado colectivamente. En su revisión de la literatura, Park \& Ungson (2001) concluyen que es más probable que la cooperación fracase que que tenga éxito a largo plazo. Por consiguiente, están interesados en los factores que conducen a la ruptura y al fracaso de una red, no en los que facilitan una cooperación exitosa del grupo. Al llamar la atención sobre lo que no se debe hacer más que lo que se debe hacer, el razonamiento que estos dos estudiosos persiguen en su modelo conceptual enriquece la literatura. Limitan la discusión a las alianzas bilaterales, pero es posible y útil aplicar sus argumentos a las redes (multi-)laterales. Park \& Ungson (2001) consideran que el principal desafío de la cooperación radica en la rivalidad entre las empresas participantes y en la complejidad de lo que se va a gobernar en medio de las incertidumbres y la vaguedad de las estrategias de gestión implicadas. Para ellos, los objetivos más importantes de la gobernanza en red son el control (el seguimiento de los costes y beneficios derivados de la cooperación entre empresas), el equilibrio de intereses y la capacidad de hacer frente a los diferentes estilos de gestión de las empresas miembro. La gobernanza se ve obstaculizada por la necesidad de reducir al mínimo sus costos a fin de evitar que se desperdicien los ahorros o los beneficios de la cooperación (Park \& Ungson, 2001). Sydow (2000) y Windeler (2001) discriminan con mayor precisión entre los objetos de la gobernanza en el contexto de lo que ellos llaman regulación de la red. Centrándose en las modalidades del marco de gobernanza para la realización de actividades dentro de las redes, 
definen seis niveles: selección, asignación, evaluación, integración de sistemas, configuración de posiciones y establecimiento de límites. Este marco representa una tipología detallada de la gama de objetos de gobernanza en la gobernanza en red. Se centra especialmente en la selección de los miembros y la delimitación de la red en relación con su entorno; la asignación de recursos y la distribución de beneficios; la definición de objetivos comunes, el control de los procesos y la evaluación de sus logros. Así pues, la regulación de la red está orientada a diversos aspectos estratégicos y operativos, y su coste total nunca debe superar el beneficio que los socios de la red obtienen de su cooperación. Los denominados coordinadores de red están a cargo de la regulación de la red, y su legitimidad les permite actuar en nombre de la red.

\section{Mecanismos de gobernanza}

El tercer elemento de un marco de gobernanza en red son los mecanismos a través de los cuales se logran realmente los objetivos de gobernanza en un contexto de acción colectiva. Convencionalmente, se distinguen dos tipos de mecanismos: mientras que las regulaciones formales estipulan órganos nominales y cargos para las personas que las hacen cumplir, los mecanismos informales de control están arraigados en instituciones sociales como la confianza, la reciprocidad o la reputación. La gobernanza informal se concibe como una gobernanza socialmente practicada, en la que los mecanismos sociales como el poder, la confianza, la simpatía y el ejercicio de la influencia se consideran instrumentos primarios de control. Esta perspectiva se basa en la integración voluntaria, que predice al menos cierto grado de familiaridad entre los socios de la red. En este caso, el control de la red es asumido por actores cuya legitimidad para esta función no se origina en oficinas legales, sino en la práctica social. Los mecanismos sociales (es decir, informales) como la confianza, la reputación, las convenciones y la formación de una cultura macro o de red, predominan como formas de coordinación en redes y guían el comportamiento económico de los actores (Jones et al., 1997; Glückler \& Armbrüster, 2003; Keast, 2016). Además, Bachmann (2001) señala la importancia del poder y la confianza como mecanismos de control capaces de afectarse mutuamente y de coordinar las acciones a varios niveles de una red.

Los estudios sobre la gobernanza formal suelen centrarse en las normas y contratos justiciables sobre los que los miembros de la red están de acuerdo por escrito. Los acuerdos de cooperación y los estatutos de asociación son ejemplos de ello. Las normas formales establecen qué derechos, obligaciones y sanciones son aplicables en la red. Varían en función de los objetivos y el contexto de la red en cuestión y van desde artículos de asociación bastante flexibles, por ejemplo, entre las prácticas médicas para mejorar la prestación de servicios sanitarios locales, hasta contratos legales vinculantes en una red de empresas conjuntas. Basándose en el concepto de estado, Schäfer (2009) se refiere a un sistema regulatorio formal dentro de las redes como una 'constitución de la red'. Las organizaciones también tienen sus propias normas que describen la estructura legal básica de su gobierno corporativo. Aunque estas instancias de gobierno corporativo pueden ayudar a regular la colaboración bilateral entre una empresa y su socio, no son eficaces para gobernar una red organizada en su conjunto y más allá de los intereses centrales de una empresa. Aquí es donde la gobernanza en red es necesaria para cerrar la brecha entre la colaboración a nivel individual (corporativo) y colectivo. Schäfer pretende mitigar el problema de la acción colectiva mediante la creación de una entidad legítima capaz de hacer cumplir las normas acordadas. El diseño formal de la gobernanza de una red aumenta así su capacidad de resolución de problemas. 
Esta conclusión subraya la necesidad de investigar la cuestión de cómo las redes organizadas pueden adquirir legitimidad e influencia. Desafortunadamente, los investigadores han estudiado hasta ahora los mecanismos de control formales e informales por separado o incluso han distinguido entre ellos como mecanismos típicos de diferentes niveles de madurez. Según los modelos de madurez de la gobernanza de redes, las redes jóvenes y relativamente pequeñas se caracterizan por mecanismos de gobernanza informal, mientras que las redes maduras y más grandes se basan en mecanismos formales como contratos, órganos y autoridades de gestión nominal (Ring \& Van De Ven, 1994; Provan \& Kenis, 2008). Como observan Provan et al. (2007), "las relaciones entre las organizaciones de una red se mantienen informalmente, a través de la estructura de la red y de las normas de reciprocidad y confianza, o formalmente, a través de la existencia de contratos, reglas y reglamentos" (p. 503). En lugar de yuxtaponer los mecanismos formales e informales como un dualismo, el análisis de las estructuras relacionales de legitimidad se centra en la simultaneidad y dualidad de las fuentes formales e informales de gobernanza lateral.

\section{Instancias de gobernanza}

El cuarto y último elemento de la gobernanza en red se refiere al lugar de control y a las entidades organizativas dotadas de autoridad legítima para gobernar. Provan y sus colegas proponen una tipología de formas de gobernanza basada en la ubicación de la autoridad formal de gobernanza (Provan \& Milward, 1995; Provan et al., 2007; Provan \& Kenis, 2008). Distinguen la auto gobernanza por los propios miembros de la gobernanza por una organización administrativa de redes $(\mathrm{NAO}=$ network administration organization). El autogobierno también puede ser ejercido centralmente por un representante autorizado de la red (un arreglo llamado gobernanza de la organización-líder) o descentralizado a través de la coordinación mutua de muchos o todos los miembros (gobernanza compartida). Esta forma descentralizada de la auto gobernanza (también llamada régimen de control lateral) se encuentra en los bufetes de abogados, las empresas consultoras y otras asociaciones (Lazega, 2000; 2001; Lazega \& Krackhardt, 2000).

La combinación de estas dos dicotomías - la autogestión frente a la gobernanza externa y la organización líder frente a la gobernanza compartida - es la base de tres formas de gobernanza, aunque no puras y exclusivas. Provan \& Kenis (2008) señalan que puede existir una NAO tanto en la gobernanza compartida como en la gobernanza de una organización líder. Además, sugieren que el rendimiento de la red depende de la elección adecuada de cada forma de gobernanza en condiciones particulares, tales como el tamaño de la red, la existencia de confianza y habilidades, y el grado de consenso sobre los objetivos entre los miembros. Si bien la auto gobernanza es adecuado para redes pequeñas con una confianza sólida entre sus miembros, la ampliación de la membresía y el aumento de la competencia de la red pueden requerir una transición a las otras dos formas de gobernanza. Otros autores, como Ring \& Van de Ven (1994), atribuyen también a las redes una trayectoria dinámica a lo largo de la cual las interacciones per se y su calidad cambian. Aparte de la útil distinción de estas formas de gobernanza, el modelo es bastante vago en cuanto a las fuentes de autoridad para ejercer el control. Mientras que Provan \& Kenis (2008) se centran en el diseño formal de las redes, cualquier autoridad de gobierno debe ser reconocida como legítima y representativa por los demás miembros para tener la capacidad de coordinar una red eficazmente (Windeler, 2001). Esta legitimidad es fundamental para el desarrollo de la teoría de la gobernanza lateral en la siguiente sección. Pocos estudios han profundizado en el significado especial que tiene el inicio de la legitimidad para el éxito de una red. 


\section{Gobernanza lateral en red}

\section{La lógica de negociación y el régimen de control lateral}

Hasta ahora, la literatura ha partido de una consideración aislada de los mecanismos de gobernanza formales e informales, sin prestar mucha atención a su interdependencia empírica. Este dualismo oculta el hecho de que las organizaciones realmente explotan ambas dimensiones de la gobernanza de la red (Lazega, 2000). Las formas de gobernanza, como el control centralizado de la red (gobernanza de la organización líder), pueden funcionar tanto formal como informalmente. Por el contrario, son concebibles las formas de gobernanza compartida contractualmente y la gobernanza informal. En la práctica, existen formas de gobernanza que no pueden calificarse de puramente informales ni formales. Por ejemplo, un código de honor, que en muchas redes corporativas se acuerda por escrito, no es informal pero no es fácilmente procesable en términos legales. El paralelismo empírico y la interdependencia de las formas formales e informales de regulación exigen, por lo tanto, una concepción alternativa de la gobernanza en red, en la que las dos dimensiones no estén aisladas sino integradas por un principio global basado en la legitimidad de la influencia y el control. Este es el objetivo que guía el concepto de gobernanza lateral.

Lazega (2001) se ha centrado en la existencia paralela de mecanismos de control formales e informales en bufetes de abogados de socios iguales. Este contexto organizacional es similar al contexto de gobernanza de las redes organizadas, que es de interés clave en este artículo. Los socios patrimoniales de un bufete de abogados, así como empresas asociados como socios en una red organizada, tienen la misma posición legal y responsabilidad por el resultado común. En su estudio empírico, Lazega (2001) encontró que en casos de conflicto los socios de un bufete de abogados tendían a evitar la intervención formal para limitar el riesgo de confrontación abierta o escalada. Al mismo tiempo, sin embargo, trataron de minimizar los costos de la gobernanza informal. De este modo, surge un régimen de control lateral a nivel colectivo (Lazega \& Krackhardt, 2000), por lo que los mecanismos formales, como las reuniones oficiales, se utilizan definitivamente como escenarios de la micropolítica informal. Aunque la orientación estratégica en este contexto está conformada por el consenso de todos los participantes, las estrategias micropolíticas y, por lo tanto, informales, tienen una gran importancia para forjar ese consenso. Basados en su estatus, prestigio y confianza, un tipo particular de actores, los llamados oligarcas figuran prominentemente en la facilitación del surgimiento del consenso. Como consecuencia, las cuestiones estratégicas de la red son discutidas por todos los involucrados, pero son moldeadas informalmente por unos pocos (Lazega, 2001). Esta percepción mejora sensiblemente nuestra comprensión de la interdependencia de los procesos formales e informales de gobernanza de la red. Son "casi imposibles de determinar o planificar desde el principio, sobre todo por el acoplamiento suelto de los elementos del sistema, y en su lugar son siempre resultados de procesos (parcialmente) autónomos que el iniciador desencadena intencionadamente pero que no puede controlar totalmente" (Sydow, 2002, p. 248).

Un acceso alternativo a la concepción de un mecanismo inclusivo de gobernanza de la red se encuentra en la lógica de acción dominante. La igualdad ante la ley y el principio de voluntariedad ordenan que todos los miembros sean considerados como personas con igual derecho a la toma de decisiones (Mayntz, 1993; Lazega, 2000). Además, todas las decisiones deben tomarse a satisfacción de los actores de la red para evitar la salida de cualquier miembro que se sienta en des- 
ventaja en contra de su voluntad y para preservar el consenso de los objetivos. Desde la perspectiva de una institucionalidad centrada en el actor en la ciencia política, Mayntz \& Scharpf (1995) postulan que la negociación es la lógica de la acción en estas situaciones. Ven la superioridad de la negociación en el hecho de que el intercambio puro conduce a efectos agregados negativos involuntarios y que el control jerárquico se rompe de todos modos porque los miembros esperan tener voz en las decisiones. La lógica de la negociación predice el interés en un resultado conjunto y facilita un acuerdo que satisfaga los deseos de todos los miembros de la red. Mientras que el principio es definitivamente practicable y ha demostrado en la teoría de juegos que es efectivo en redes pequeñas, el consenso de metas y la toma de decisiones se vuelven cada vez más difíciles a medida que aumenta el tamaño del grupo (Scharpf, 2006). La eficacia y la eficiencia de la toma de decisiones no es lo único que sufre en los grandes grupos. También existe el creciente peligro de que las minorías sean derrotadas y de que la cohesión disminuya. En línea con Provan \& Kenis (2008), por lo tanto, la gobernanza compartida se vuelve problemática más allá de un cierto tamaño de red (Mayntz, 1993).

\section{El concepto de la gobernanza lateral en red}

¿Cómo se puede entonces lograr la gobernanza legítima de redes relativamente grandes? Para responder a esta pregunta, es útil examinar otros ámbitos de la sociedad que se enfrentan a problemas similares. Quizás el caso más instructivo sea la discusión de los sistemas democráticos en la teoría política. En las democracias, en las que por definición no existen directrices jerárquicas, las decisiones deben tomarse entre pares independientes, al igual que en las redes. Para que las decisiones colectivas sean viables, las soluciones varían en un continuo entre formas simétricas y asimétricas de forjar acuerdos. En los enfoques simétricos, el voto de cada individuo tiene igual peso en la decisión del grupo, mientras que en los enfoques asimétricos, la autoridad discrecional se transfiere a uno o unos pocos representantes para tomar decisiones vinculantes para todos.

Entre estos dos extremos puede haber esquemas que definan mayorías específicas, cada uno de los cuales tiene sus méritos e inconvenientes. Mientras que los procedimientos asimétricos ofrecen la ventaja de una rápida toma de decisiones, sufren el peligro del boicot de las minorías. Por el contrario, los procedimientos simétricos prometen decisiones basadas en el consenso, pero las negociaciones necesarias a las que se refiere Mayntz (1993) pueden prolongarse indefinidamente. Para resolver este dilema, los sistemas democráticos delegan el poder de decisión, concentrándolo en un pequeño grupo de individuos que representan los intereses de su electorado y gozan de autorización para negociar decisiones aplicables a todos. De este modo, las decisiones pueden ser tomadas legítima y eficazmente por un pequeño número de personas. A pesar de todas las diferencias entre un sistema burocrático público y las redes organizadas privadamente, el principio de delegación de poder ofrece un punto de partida prometedor para responder a la pregunta de cómo abordar las cuestiones de gobernanza en las redes.

Supongo que el éxito de la coordinación en las redes organizadas se basa en el principio de la delegación del poder de decisión. Esta delegación es un requisito previo y un origen principal del concepto de gobernanza lateral en red, en el que todos los miembros comparten legítimamente. La perspectiva de la gobernanza lateral en red puede situarse dentro de los cuatro elementos del marco de gobernanza explicado anteriormente. En primer lugar, hace justicia a un contexto de gobernanza en el que los miembros tienen el mismo derecho a participar. En segundo lugar, puede aplicarse a cualquier objeto de gobernanza dentro de una red que persiga objetivos comunes 
o bienes colectivos. En tercer lugar, la gobernanza lateral se basa en una forma de legitimación que puede arraigarse tanto formal como informalmente, y estos dos mecanismos pueden ser integrados y observados simultáneamente.

La concentración en la intensidad y el intercambio de legitimidad en una red supera el dualismo de la gobernanza de redes formales e informales, tanto teórica como empíricamente. Esta perspectiva es apoyada por Human \& Provan (2000), quienes también enfatizan la importancia de la legitimidad de los actores, las acciones y las estructuras, sin separar sus causas en aspectos informales y formales. Además, Weber (1978) también señala que la legitimidad puede provenir de prácticas informales como la tradición, los sentimientos o los valores, así como de sistemas de legalidad formalmente aceptados. Por lo tanto, el enfoque adoptado aquí se abstiene de discriminar entre fuentes formales e informales de legitimidad y pretende evaluar la distribución relacional de la legitimidad entre los agentes de gobierno dentro de una red organizada. En cuarto lugar, y en consecuencia, el agente de la gobernanza no se explica en términos de aspectos formales o informales, sino más bien por su grado de legitimidad. Por lo tanto, la delegación del poder legítimo de decisión satisface teóricamente las dos exigencias de la gobernanza en red mencionadas anteriormente: la coordinación basada en una asociación de iguales y la minimización de los costes de transacción.

\section{Agentes legítimos locales y globales de gobernanza}

La delegación legítima de la toma de decisiones puede distribuirse entre varios grupos de interés de la red. Dependiendo de la estructura relacional de los grupos de intereses, pueden surgir subgrupos que conjuntamente se otorguen una representación legítima de sus intereses y que, en su mutualidad, puedan diferenciarse claramente de otros subgrupos. Estos subgrupos estructuran la red en lo que yo llamo facciones legítimas. La figura 1 muestra una distribución hipotética que describe la transferencia de la autoridad legítima para la toma de decisiones entre los miembros de una red. En este modelo, cada uno de los cuatro miembros tiene al menos tres votos de la red y posee la mayor legitimidad, medida por el número de veces que ha sido nombrado. Sin embargo, los tipos de miembros A y B difieren en la estructura de esos votos. Los votos para el tipo A provienen todos de una facción local; los votos para el tipo B, de todas las facciones de la red. Mientras que los miembros de tipo A son localmente legítimos, los miembros de tipo B son globalmente legítimos. Este ejemplo ilustra la importancia que tiene la estructura específica de legitimidad cuando sus colegas confieren a los miembros individuales de una red grados comparables de legitimidad. Si un miembro recibe muchos votos pero exclusivamente de una facción (tipo A), entonces esa persona tiene legitimidad local. Por el contrario, la legitimidad global permite a un miembro contribuir mucho más a las decisiones aceptables en toda la red. En lo que respecta a la lógica de acción de una red, los actores que gozan de ese tipo de legitimidad (tipo B) pueden entablar negociaciones y, por lo tanto, tomar decisiones colectivas con mayor facilidad que toda la red. Por supuesto, el que los actores de tipo B logren obtener el consentimiento depende en última instancia de la gravedad del conflicto entre las facciones (tal como lo mantienen sus representantes de tipo A) y de la legitimidad y la capacidad de los actores de tipo B para mediar en sus intereses $y$, en última instancia, reconciliar su conflicto. ${ }^{2}$

Los actores de tipo A representan exclusivamente los intereses de sus propias facciones y, por lo tanto, entrarán en negociación con una clara participación. Por el contrario, a medida que los actores de tipo B obtienen legitimidad de varias facciones, pueden correr el riesgo de perder 
Figura 1:

Red hipotética de transferencia legítima de la autoridad decisoria

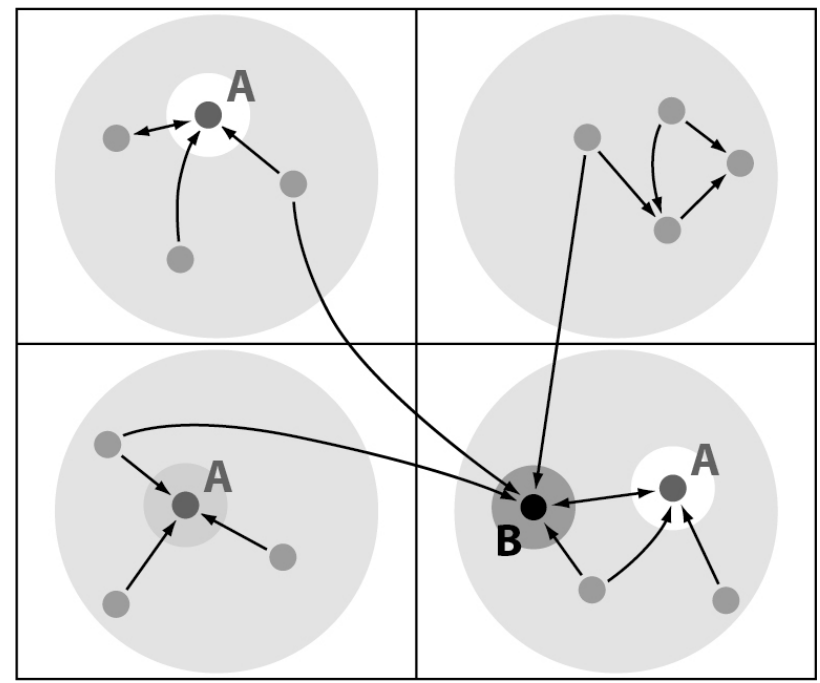

La gobernanza eficiente en red depende de una distribución particular de la legitimidad en la red. Las distribuciones relacionales del poder legítimo de decisión varían con la magnitud y la fuente del poder de decisión delegado. Un análisis empírico de las estructuras de gobernanza se centra, pues, en las siguientes cuestiones de investigación: En primer lugar, y metodológicamente, ¿cómo se pueden evaluar las estructuras relacionales de legitimidad? En segundo lugar, y empíricamente, ¿cómo se superponen los patrones observados de las relaciones legítimas de gobernanza (la distribución empírica de la legitimidad) con las estructuras formales de gobernanza en una red organizada o se apartan de ellas? En tercer lugar, y de manera efectiva, ¿cómo afecta una distribución empírica de la delegación legítima de autoridad al resultado de la red? Estas cuestiones se abordarán en un estudio de caso comparativo de dos redes organizadas de pequeñas y medianas empresas en Alemania.

\section{Diseño de investigación}

\section{Dos redes organizadas: consultoría y tecnología dental}

El primer estudio de caso fue sobre ConsultingNet, ${ }^{3}$ una red regional de 23 consultores corporativos que operaban principalmente para seguir el intercambio profesional entre sus miembros por medio de eventos y reuniones regulares. ConsultingNet era una asociación registrada cuya estructura de gobierno estaba escrita en sus estatutos. El consejo de administración estaba compuesto por dos miembros y era responsable de la gestión de las actividades de la red. El límite de la asociación en cuanto a la duración del mandato y la elección de los dos miembros durante la

parte o toda esa legitimidad en casos de conflicto si las facciones individuales sienten que sus intereses no están suficientemente apoyados. Por lo tanto, una posición de tipo B per se no es una condición suficiente para resolver conflictos dentro de la red.

ConsultingNet es un seudónimo 
asamblea general anual podría decirse que hace de la junta un agente formal de gobierno bastante débil. A pesar de las normas establecidas en esos documentos, en el plano jurídico se preveía relativamente poco la influencia y la división del trabajo en una asociación, sobre todo si, como en este caso, no se había resuelto el recurso a las sanciones. Un miembro que quebrantara el código de conducta podría ser expulsado de la asociación, pero no se habían formalizado otras formas menos drásticas de sancionar la conducta. El modelo de gobernanza dentro de la red ConsultingNet es, por tanto, el más parecido al modelo de gobernanza compartida (Provan \& Kenis, 2008).

El segundo estudio de caso fue sobre Dentis, ${ }^{4}$ una red de 27 laboratorios dentales pequeños y medianos en toda Alemania. Dentis era una sociedad de responsabilidad limitada cuyos estatutos estipulan la elección de cinco representantes de la empresa para el consejo de supervisión en la junta de accionistas. La responsabilidad de las operaciones de la red recae en una oficina de gestión, que funciona como NAO (Human \& Provan, 2000). Debido a la separación geográfica de las firmas miembro y a la diferente, y en algunos casos intensa, cooperación multilateral entre ellas, la red celebró reuniones trimestrales de accionistas, en las que el Consejero Delegado, los miembros del Consejo de Supervisión y los accionistas negocian, deciden y evalúan las actividades conjuntas. Las altas cuotas de membresía para los individuos y la notable cantidad de trabajo y tiempo invertido caracterizan a Dentis como una red muy activa y a largo plazo. Los objetivos de Dentis eran lograr una producción en red y buscar el desarrollo conjunto de nuevos conceptos y soluciones en marketing, distribución, Tl, estándares de calidad, capacitación y otras áreas. La estructura de gobierno de Dentis tipifica el gobierno compartido con una NAO operada conjuntamente (Provan \& Kenis, 2008).

\section{Cuadro 1:}

Gobernanza formal de dos redes corporativas

\begin{tabular}{|l|l|l|}
\hline Elementos & ConsultingNet & Dentis \\
\hline Form jurídica & Asociación registrada & Sociedad Limitada \\
\hline Mecanismo de control & Junta directiva & NAO*, consejo supervisor \\
\hline Instancia de gobernanza & Asamblea general de socios & Junta General de Accionistas \\
\hline Modelo de gobernanza & Gobernanza compartida & Gobernanza compartida, NAO \\
\hline
\end{tabular}

*Network administration organization.

Ambas redes organizadas fueron investigadas según el procedimiento de investigación SONA - análisis situacional de redes organizacionales (Glückler \& Hammer, 2015) y evaluadas durante un período prolongado. SONA hace uso de la observación cualitativa durante las entrevistas personales y grupales, así como de los datos cuantitativos recopilados con una encuesta estandarizada de redes y evaluados con métodos de análisis de redes sociales. El siguiente análisis de la estructura de gobernanza se basa en numerosas entrevistas y, para cada red, en una encuesta que cubre más del $70 \%$ de los miembros (véase el cuadro 2). 
Cuadro 2:

SONA: Diseño de investigación, método mixtos y colección de datos

\begin{tabular}{|l|r|r|}
\hline Instrumento & ConsultingNet & Dentis \\
\hline Discusión prelminiar & 1 & 1 \\
\hline Número de entrevistas & 4 & 5 \\
\hline Repuesta a la encuesta (tasa) & $19(82,6 \%)$ & $20(74,1 \%)$ \\
\hline Discusión en grupo (validación) & 1 & 1 \\
\hline Número de miembros de la red & 23 & 27 \\
\hline
\end{tabular}

Las dos redes encajan en la anterior clasificación de la gobernanza de la red. El contexto era el mismo para ambos. Por supuesto, la situación del mercado varía de una firma miembro a otra porque cada una de las firmas está afiliada a un sector económico diferente, pero en ambas redes las compañías que son al menos rivales potenciales se han unido para mejorar su competitividad. Las firmas miembro de las dos redes eran legalmente autónomas y económicamente independientes entre sí. En cuanto al objeto de la gobernanza, la divergencia entre las redes era mayor por lo que hacían. Mientras que ConsultingNet se dedicaba a actividades bastante blandas que no requerían un uso intensivo de capital para sus miembros (por ejemplo, eventos), Dentis perseguía objetivos más ambiciosos de desarrollo colectivo e inversión en producción en red y estrategias conjuntas de marketing y ventas. La consiguiente complejidad de la gobernanza en esa red quedó de manifiesto en el hecho de que se utilizara una Oficina Nacional de Auditoría. Las redes también tenían mecanismos de gobernanza y agentes de gobernanza similares. Sus reglamentos eran formales, al igual que las autoridades formales concomitantes de la gobernanza. En ambas redes, sin embargo, las entrevistas personales indicaron la conciencia de una dimensión paralela de control que no estaba formalmente regulada, sin la cual ninguna de las dos habría sido viable.

\section{Medición de la delegación legítima de la autoridad decisional}

Las discusiones preliminares, así como las entrevistas personales con los miembros de las dos redes, mostraron que la gobernanza de las redes estaba repetidamente vinculada a situaciones críticas de toma de decisiones a nivel de la red. A efectos de observación empírica, la delegación del poder de decisión entre los miembros de una red organizada se operacionalizó con la siguiente afirmación y pregunta:

"Imaginen que una decisión importante debe ser tomada en la red y que no pueden participar en ella. ¿Qué otros miembros de la red tendrían que estar presentes cuando se tome la decisión para que usted pueda aceptar el resultado?"

Este punto de la pregunta facilita una medición independiente de la legitimidad de cada actor como agente de gobernanza, ya que la evaluación multilateral de un miembro por otros no puede ser afectada por ese miembro en el momento de la encuesta. Aunque las preguntas hipotéticas son más discutibles que las preguntas destinadas a reconstruir acontecimientos reales, los estudios instructivos de Lazega (2000, 2001), por ejemplo, prueban que las preguntas hipotéticas pueden efectivamente capturar representaciones subjetivas válidas de las estructuras sociales de influencia. Así pues, una encuesta de la red recoge la estructura completa de todos los votos 
sobre la delegación de autoridad para tomar decisiones. Para caracterizar la distribución de legitimidad resultante, dos parámetros son importantes: la magnitud y la estructura de la legitimidad dentro de la red.

Magnitud. La magnitud de la legitimidad se mide por el grado de entrada. El grado $d_{1}$ de un miembro $n$ es el número de votos (menciones) $v$ que un miembro recibe de los otros miembros $j$ para que pueda participar en las decisiones como representante legítimo en su ausencia.: $d_{l}\left(n_{i}\right)=\sum_{j} v_{i j}$. El máximo grado de entrada para cada red de tamaño $N$ es $n-1$.

Fuente. La dimensión estructural se refiere a la distribución específica de la transferencia de la autoridad de toma de decisiones. Una fuerte legitimidad puede descansar en los votos de una facción unitaria o en los votos de los miembros de varias facciones. Para conocer la distribución específica de las transferencias de toma de decisiones, utilizo el índice externo-interno (E-I) (Krackhardt \& Stern, 1988). Mide la relación entre las relaciones de legitimidad entre las facciones (legitimidad externa o $E L$ ) y las relaciones de legitimidad dentro de las facciones (legitimidad interna o $I L)$. El índice $E-I$ se calcula de la siguiente manera: $E-I=(E L-I L) \div(E L+I L)$. Sin embargo, como la $E-I$ sólo puede calcularse en redes simétricas, no se puede prescindir de la dirección de la votación entre los miembros. Por lo tanto, la E-I no indica si una transferencia de autoridad para la toma de decisiones va de $\mathrm{A}$ a $\mathrm{B}$ o más bien de $\mathrm{B}$ a $\mathrm{A}$. Calcula sólo el grado en que cada miembro está involucrado en las relaciones de legitimidad internas o externas de las facciones. Teóricamente, la $E-l$ varía entre -1 (únicamente relaciones entre un facción interna) y +1 (únicamente relaciones con otras facciones externas). ${ }^{5}$

La magnitud y la fuente de la distribución de la legitimidad se utilizan como dimensiones de un modelo de gobernanza de red lateral. Juntos, producen una matriz con cuatro cuadrantes subdivididos por el valor medio del grado y el valor nulo del índice E-I. El modelo permite la definición formal de tres tipos de legitimidad de una persona (Figura 2):

Figura 2:

Modelo de gobernanza lateral: transferencia legítima de la autoridad decisoria

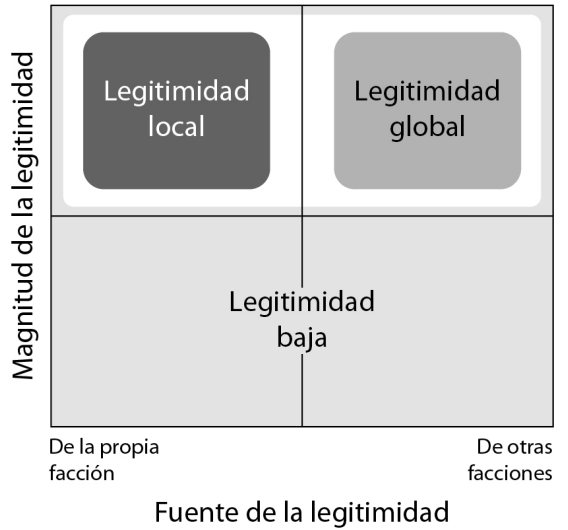

Los valores del índice E-I que se acercan a +1 no son realistas porque el análisis fraccional previo de la red de legitimidad clasifica a los miembros en facciones coherentes basadas en la red. Por lo tanto, la afiliación fraccional hace que la tendencia hacia las relaciones internas de las facciones sea probable. 
Legitimidad baja. Los miembros con una titulación inferior a la media tienen menos probabilidades que los miembros con una titulación superior a la media de obtener una aceptación suficiente de sus decisiones en la red.

Legitimidad local. Los miembros del cuadrante superior izquierdo tienen una legitimidad superior a la media, pero proviene en su mayoría de su propia facción. Al igual que la cabeza de una facción, pueden ganar aceptación para las decisiones dentro de sus facciones.

Legitimidad global. Los miembros del cuadrante superior derecho tienen una legitimidad superior a la media procedente de diversas facciones. Hablando en nombre de una red, estas personas tienen la mayor oportunidad de ganar la aceptación de las decisiones en toda la red.

En consecuencia, el objetivo de analizar empíricamente la estructura de distribución de la legitimidad en las redes ConsultingNet y Dentis era determinar en qué medida esa estructura se corresponde con el modelo formal de gobernanza y en qué medida creó legitimidad general.

\section{Estructuras empíricas de la gobernanza lateral en las redes}

\section{Gobernanza planificada versus gobernanza practicada}

Debido al principio democrático de la delegación de poder, la estructura de gobierno formalmente planificada no tiene por qué estar a la altura de la distribución real de la legitimidad en la red. Los cargos formales, como los de director gerente o miembro del consejo de administración, y los órganos formales, como el consejo asesor o de supervisión, establecen las responsabilidades y la autoridad para tomar decisiones, pero no excluyen la legitimidad de otros miembros de la red. Algunos miembros pueden ser vistos como importantes (legítimos) para ciertas decisiones en la red, aunque no tengan un cargo que les otorgue legalmente la autoridad para tomar decisiones. En cambio, la iniciativa e influencia de otros miembros en el proceso de toma de decisiones puede incluso ser esperada o requerida.

Para probar esta suposición, comparamos las formas de gobierno de la red formalmente planificadas y realmente practicadas. La Figura 3 yuxtapone el diagrama de tipo ideal de gobernanza formal con las distribuciones de legitimidad empíricamente documentadas. La estructura de distribución de la legitimidad en las dos redes está representada por nodos que representan a los miembros de las respectivas redes y por bordes que representan las relaciones individuales entre los miembros encuestados que participan en la delegación de la autoridad legítima para la toma de decisiones.

En ambos estudios de caso sólo existe una relación estadística moderada entre la gobernanza planificada y la practicada (con coeficientes de 0,18 en el caso de ConsultingNet y 0,23 en el caso de Dentis). Este resultado confirma la expectativa y, desde la perspectiva de las redes, la esperanza de que la estructura planificada de gobernanza esté contenida en la distribución real de la legitimidad: Los cargos formales son ocupados por actores legítimos. Sin embargo, la distribución real de la legitimidad se desvía de la estructura planificada de manera notable, como lo 
revelan los dos parámetros que miden esta faceta de las redes, la densidad y la centralización. La densidad de la red muestra el porcentaje de los votos teóricamente posibles que se recibieron. La centralización mide la tendencia de la red a concentrar todas las relaciones de delegación en un solo actor. Unanimidad - 100\% de centralización - se registraría como una estrella perfecta. Si las relaciones de delegación se distribuyeran completamente entre los miembros, la centralización sería $\mathrm{O}$. El análisis muestra que la gobernanza practicada en ambas redes se distribuye entre muchos más miembros de lo que se había previsto formalmente. Las estructuras que se utilizaron realmente tenían una densidad superior a la prevista, y el grado de delegación de poderes estaba menos centralizado.

Figure 3:

Una comparación entre la gobernanza planificada y la practicada en dos redes

ConsultingNet
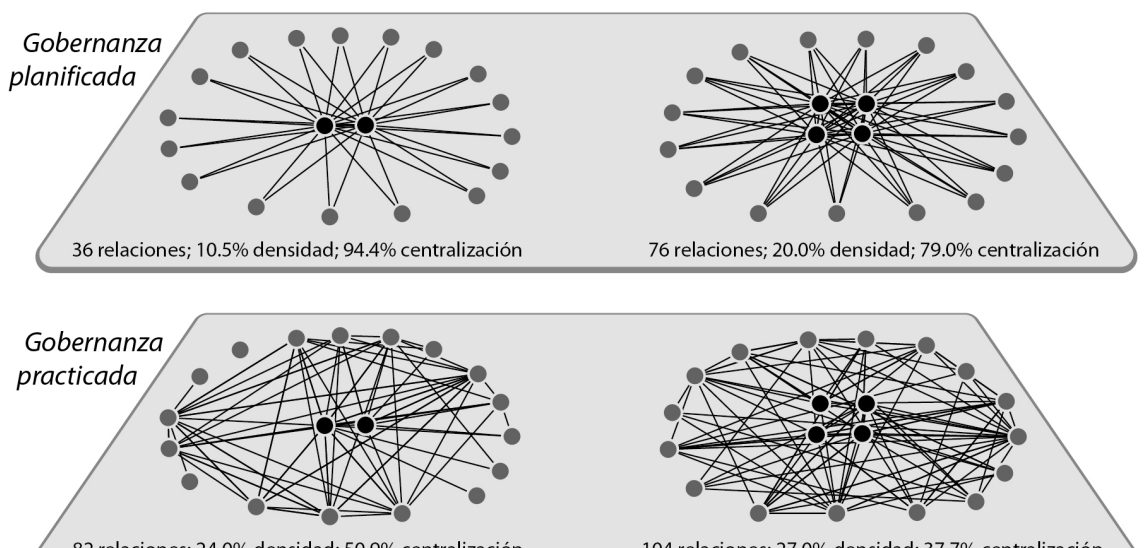

82 relaciones; $24.0 \%$ densidad; $50.9 \%$ centralización
Dentis

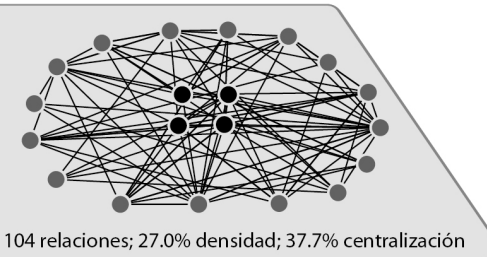

Nodos grises = miembros de la red; nodos negros = miembros de la red en el consejo de administración

Por lo tanto, el número real de actores legítimamente posicionados para afectar el proceso de toma de decisiones aceptables excede sustancialmente el número estipulado. El bajo grado de centralización con la gobernanza tal como se practica en la práctica refleja la tendencia de la gobernanza lateral a no reducirse a las instancias formales de la gobernanza. En virtud de la legitimidad social, los miembros informales también figuran en la gobernanza tal como se practica en las redes, convirtiéndose en parte del agente de facto de la gobernanza de las redes. El modelado de una estructura de gobernanza formal no capta la realidad de la gobernanza de la red. Por lo tanto, centrarse únicamente en la composición formal de las juntas ejecutivas puede resultar rápidamente inadecuado. Los miembros legítimos pueden sentirse desatendidos o pueden impugnar posteriormente las decisiones que se han tomado.

\section{Legitimidad local versus global: Estructuras para la transferencia de la autoridad decisoria}

La primera parte del análisis mostró la divergencia entre la gobernanza practicada y la planificada en las redes laterales. Sin embargo, el concepto de gobernanza lateral en red permite una evaluación aún más discernida de las estructuras expresas de la distribución de la legitimidad. 
En esta sección utilizo el modelo de gobernanza de red lateral desarrollado anteriormente, que permite establecer una matriz en la que se representa la legitimidad de cada miembro de la red en función de su magnitud (grado de entrada) y estructura (índice E-I). La matriz ayuda a distinguir tres roles de agencia: bajo, local y global. El papel de la baja legitimidad se caracteriza por el grado marginal de legitimidad conferido por los socios de la red (Figura 4, cuadrantes inferior izquierdo y derecho). La baja legitimidad se registra como un grado por debajo del promedio ( ), que refleja el número de veces que un actor de la red es nombrado por otros miembros.

Figure 4:

Distribución empírca de legitimidad en la matriz de gobernanza lateral en red

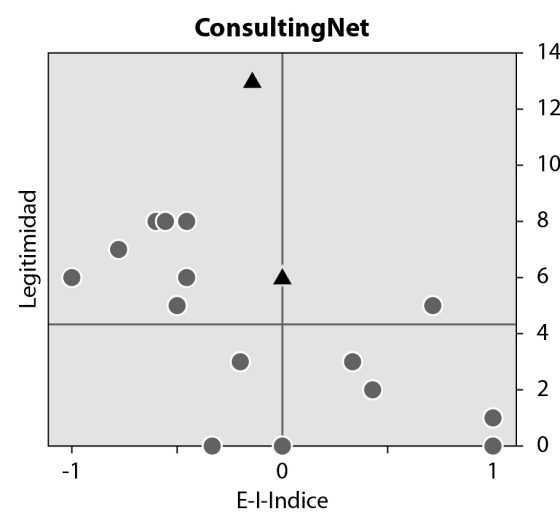

A Comité ejecutivo/consejo de vigilancia Socio

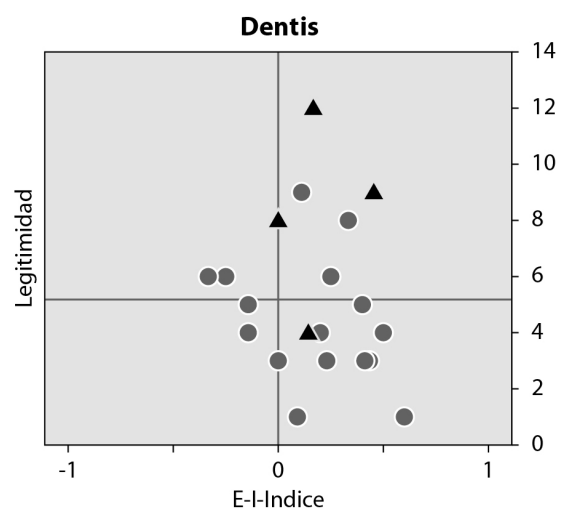

A Comité ejecutivo/consejo de vigilancia Socio

Todos los miembros con una legitimidad superior a la media están representados en los cuadrantes superiores de la matriz y son el agente analítico de la gobernanza. Son muy relevantes para la gobernanza, pero difieren -algunos de ellos considerablemente- en su posición dentro de la estructura distributiva a través de la cual se otorga la autoridad legítima para la toma de decisiones. El segundo papel de la legitimidad -el local- aparece en los cuadrantes superiores izquierdos (Figura 4). Su legitimidad por encima de la media es otorgada por un subgrupo más o menos unitario que puede describirse como una facción porque tiene un $E-I$ menor que cero en cada caso. En contraste, el tercer papel de la legitimidad, el global, aparece en el cuadrante superior derecho (Figura 4). Se caracteriza por una E-I superior a cero, tiene su legitimidad desde la red en su conjunto, es decir, también desde fuera de una facción unitaria. Los miembros con legitimidad local, como en ConsultingNet, abogan por decisiones para una parte homogénea de la red, y esas decisiones no necesariamente gozan de apoyo en toda la red. Los miembros con legitimidad global, sin embargo, se unen por encima del promedio y la aceptación de toda la red por parte de los demás miembros y tienen una mayor probabilidad de ser capaces de trabajar hacia decisiones que son más capaces de crear consenso y ganar aceptación de lo que sería el caso de otra manera. Estos miembros sirven como representantes de la red en su conjunto, como en la red Dentis.

La Figura 4 ilustra la distribución de los miembros en la matriz para ambas redes. Los modelos empíricos sugieren tres puntos de vista importantes. En primer lugar, ambas redes tienen un agente de gobierno que no está adecuadamente representado por sus oficinas formales. En ambas redes, la autoridad para tomar decisiones por encima de la media se delega en un puñado 
de miembros cuya legitimación es considerablemente mayor que la puramente formal. En la ConsultingNet, además de los dos miembros de la junta formalmente elegidos, siete miembros más fueron nombrados como representantes legítimos. En Dentis, otros cuatro fueron considerados igualmente legítimos que los cuatro miembros formales de la junta. En segundo lugar, el lugar que ocupan las oficinas formales en ambas redes es evidente. Aunque existen similitudes significativas entre las estructuras de legitimación planificadas y las que se practican en la actualidad, ambas redes tenían muchos otros miembros con una legitimidad superior a la media. Por lo tanto, el agente identificado de la gobernanza lateral de la red delinea la estructura de gobernanza real y complementa el trabajo del consejo de supervisión.

En tercer lugar, ConsultingNet y Dentis difieren considerablemente en la composición de las dos funciones de gobernanza. Los miembros de ConsultingNet con una legitimidad superior a la media derivaron su aceptación casi exclusivamente de una facción local, no de la red en su conjunto. Por el contrario, la distribución de la legitimidad en Dentis reveló que muchos miembros con una legitimidad superior a la media en toda la red y que, por lo tanto, podrían ganar aceptación en la red mucho más fácilmente que los miembros prominentes de ConsultingNet con legitimidad sólo local. En particular, ConsultingNet no estaba presente en el momento del análisis de los datos de la encuesta, mientras que Dentis siguió invirtiendo en la profesionalización. Dando razón de los problemas citados en el estudio de caso de ConsultingNet, sus miembros describieron asuntos que afectaban a toda la red y que habían suscitado un torrente de objeciones. Este hecho indica que la legitimidad de los responsables de la toma de decisiones sólo había sido parcial o insuficiente. Mientras tanto, una gran parte de los miembros de la red la habían abandonado. Por el contrario, el odontólogo no ha tenido problemas serios con su gobernabilidad y capacidad de acción y ha seguido cumpliendo con éxito sus objetivos comunes.

\section{Conclusión}

Este artículo ha elaborado una teoría de la gobernanza lateral en red. Conceptualmente, supera la brecha entre la gobernanza formal e informal al centrarse en la distribución relacional de la legitimidad para gobernar y controlar en un acuerdo de gobernanza entre iguales. El concepto de gobernanza lateral en red trata de conciliar el carácter voluntario de la pertenencia a la red y las expectativas de cooperación no jerárquica, por una parte, con el interés en agentes eficaces de gobernanza y el cumplimiento de sus normas normativas y de procedimiento, por otra. Si bien este artículo se ha centrado predominantemente en la legitimidad, la investigación futura debería explorar más a fondo el papel del poder en las redes de gobernanza. El nexo con los conceptos de poder constituye un vínculo prometedor entre la geografía económica y la geografía política (Allen \& Cochrane, 2007; Allen, 2011; Griffin, 2012; Grabs \& Ponte, 2019).

Metodológicamente, me he basado en métodos de análisis de redes sociales para convertir este concepto en una práctica empírica. Si bien los métodos de redes se han ido reconociendo y utilizando cada vez más en la geografía humana (Giuliani, 2007; Ter Wal \& Boschma, 2009; Glückler, 2013; Glückler \& Hoffmann, 2019), al mismo tiempo, los estudios de redes han reconocido el papel del espacio y el lugar en las redes sociales reales (Doreian \& Conti, 2012). La investigación futura sobre la gobernanza territorial se beneficiará de un mayor intercambio entre los estudios 
de redes y la geografía, por ejemplo, adoptando enfoques posicionales, dinámicos y multiniveles (Glückler \& Doreian, 2016; Lazega \& Snijders, 2016; Glückler et al., 2017).

Empíricamente, el análisis de dos estudios de caso señala que concentrarse únicamente en la estructura formal de la gobernanza y descuidar las esferas invisibles de la gobernanza realmente practicada puede conducir a conflictos y a una creciente resistencia en la colaboración en red. En cambio, el análisis de la distribución relacional de la legitimidad ayuda a examinar y, si es necesario, adaptar la gobernanza de la red, identificando a los actores más legítimos y discriminando el lugar - local o global - de su legitimidad para la gobernanza.

Una teoría de la gobernanza lateral en red no implica arquitecturas estáticas de la organización, sino que sirve como una brújula que guía la gobernanza diaria de la red. El diseño real de una estructura de gobernanza no sólo depende de los cuatro componentes básicos - contexto, objeto, mecanismo y agencia de gobernanza - sino también de su contexto geográfico e institucional para transmitir una práctica apropiada, legítima y eficaz de gobernanza en red.

\section{Agradecimientos}

Este artículo se basa en una investigación que se ha realizado en parte en colaboración con Schila Nemeth e Ingmar Hammer. Les estoy agradecido por su valioso apoyo en la recopilación y análisis de los datos de los estudios de caso y en la discusión de los conceptos desarrollados aquí. Se agradece el apoyo financiero del Ministerio Alemán de Educación e Investigación (BMBF).

\section{Acknowledgements}

This article draws on research that has partly been realized in collaboration with Schila Nemeth and Ingmar Hammer. I am grateful to them for their valuable support in collecting and analyzing the data of the case studies and in discussing the concepts developed here. Financial support by the German Ministry of Education and Research (BMBF) is gratefully acknowledged.

\section{Referencias}

ALLEN, J. Topological twists: Power's shifting geographies. Dialogues in Human Geography, 2011, 1 (3): 283-298.

ALLEN, J. \& COCHRANE, A. Beyond the territorial fix: Regional assemblages, politics and power. Regional Studies, 2007, 41 (9): 1161-1175.

ANSELL, C. \& GASH, A. Collaborative governance in theory and practice. Journal of Public Administration Research \& Theory, 2008, 18 (4): 543-571.

ANSELL, C. \& TORFING, J. (editores) Handbook on Theories of Governance. Cheltenham, UK: Edward Elgar, 2016. 
BACHMANN, R. Trust, power and control in trans-organizational relations. Organization Studies, 2001, 22: 337-365.

BULKELEY, H. Reconfiguring environmental governance: Towards a politics of scales and networks. Political Geography, 2005, 24 (8): 875-902.

CHARRON, N., DIJKSTRA, L. \& LAPUENTE, V. Regional governance matters: Quality of government within European Union member states. Regional Studies, 2014, 48 (1): 68-90.

COE, N. M., DICKEN, P. \& HESS, M. Global production networks: Realizing the potential. Journal of Economic Geography, 2008, 8 (3): 271-295.

CRANG, M., HUGHES, A., GREGSON, N., NORRIS, L. \& AHAMED, F. Rethinking governance and value in commodity chains through global recycling networks. Transactions of the Institute of British Geographers, 2013, 38 (1): 12-24.

CROUCH, C. Capitalist Diversity and Change: Recombinant Governance and Institutional Entrepreneurs. Oxford: Oxford University Press, 2005.

DOREIAN, P. \& CONTI, N. Social context, spatial structure and social network structure. Social Networks, 2012, 34 (1): 32-46.

FEIOCK, R. C. Rational choice and regional governance. Journal of Urban Affairs, 2007, 29 (1): 4763.

GEREFFI, G., HUMPHREY, J. \& STURGEON, T. The governance of global value chains. Review of International Political Economy, 2005, 12 (1): 78-104.

GIBSON-GRAHAM, J. K. Diverse economies: performative practices for ,other worlds'. Progress in Human Geography, 2008, 32 (5): 613-632.

GIULIANI, E. The selective nature of knowledge networks in clusters: Evidence from the wine industry. Journal of Economic Geography, 2007, 7 (2): 139-168.

GLÜCKLER, J. Organisierte Unternehmensnetzwerke: Eine Einführung. In: GLÜCKLER, J., DEHNING, W., JANNECK, M. \& ARMBRÜSTER, T. (editores). Unternehmensnetzwerke. Architekturen, Strukturen und Strategien. Heidelberg: Springer Gabler, 2012, p. 1-18.

GLÜCKLER, J. Geografía económica y evolución de redes. In: VALDIVIA LÓPEZ, M. \& DELGADILLO MACÍAS, J. (editores). La Geografía y la Economía en sus Vínculos Actuales: una Antología Comentada del Debate Contemporáneo. Ciudad Universitaria, delegación Coyoacán, México, D.F.: Universidad Nacional Autónoma de México, Instituto de Investigaciones Económicas, 2013, p. 539-556.

GLÜCKLER, J. \& ARMBRÜSTER, T. Bridging uncertainty in management consulting: The mechanisms of trust and networked reputation. Organization Studies, 2003, 24 (2): 269-297. 
GLÜCKLER, J., DEHNING, W., JANNECK, M. \& ARMBRÜSTER, T. (editores) Unternehmensnetzwerke. Architekturen, Strukturen und Strategien. Heidelberg: Springer Gabler, 2012.

GLÜCKLER, J. \& DOREIAN, P. Editorial: Social network analysis and economic geography - positional, evolutionary and multi-level approaches. Journal of Economic Geography, 2016, 16 (6): 1123-1134.

GLÜCKLER, J. \& HAMMER, I. Cooperation gains and network goods. In: JUNG, S., KREBS, P. \& TEUBNER, G. (editores). Business Networks Reloaded. Surrey, UK: Ashgate, 2015, p. 22-41.

GLÜCKLER, J. \& HOFFMANN, J. Prácticas económicas alternativas y análisis de redes sociales. In: SÁNCHEZ HERNÁNDEZ, J. L. (editor) Espacios y Prácticas Económicas Alternativas en las Ciudades Españolas. Cizur Menor, Navarra: Thomson Reuters Aranzadi, 2019, p. 237-249.

GLÜCKLER, J., LAZEGA, E. \& HAMMER, I. (editores) Knowledge and Networks. Knowledge and Space Series, Band 11. Cham: Springer International, 2017.

GLÜCKLER, J. \& PANITZ, R. Relational upgrading in global value networks. Journal of Economic Geography, 2016, 16 (6): 1161-1185.

GRABS, J. \& PONTE, S. The evolution of power in the global coffee value chain and production network. Journal of Economic Geography, 2019.

GRIFFIN, L. Where is power in governance? Why geography matters in the theory of governance. Political Studies Review, 2012, 10: 208-220.

HARDIN, G. The tragedy of the commons. Science, 1968, 162: 1243-1248.

HUMAN, S. E. \& PROVAN, K. G. Legitimacy building in the evolution of small-firm multilateral networks: A comparative study of success and demise. Administrative Science Quarterly, 2000, 45 (2): 327-365.

JONES, C., HESTERLY, W. S. \& BORGATTI, S. P. A general theory of network governance: Exchange conditions and social mechanisms. Academy of Management Review, 1997, 22: 911-945.

JUNG, S., KREBS, P. \& TEUBNER, G. (editores) Business Networks Reloaded. Surrey, UK: Ashgate, 2015.

KEAST, R. Network governance. In: ANSELL, C. \& TORFING, J. (editores). Handbook on Theories of Governance. Cheltenham, UK: Edward Elgar, 2016, p. 442-453.

KRACKHARDT, D. \& STERN, R. N. Informal networks and organizational crises: An experimental simulation. Social Psychology Quarterly, 1988, 51 (2): 123-140.

LAZEGA, E. Rule enforcement among peers: A lateral control regime. Organization Studies, 2000, 21 (1): 193-214. 
LAZEGA, E. The Collegial Phenomen. Oxford: Oxford University Press, 2001.

LAZEGA, E. \& KRACKHARDT, D. Spreading and shifting costs of lateral control in a law partnership: A structure analysis at the individual level. Quality and Quantity, 2000, 34: 153-175.

LAZEGA, E. \& SNIJDERS, T. (editores) Multilevel Network Analysis for the Social Sciences. Theory, Methods and Applications. Methodos Series, Band 12. Heidelberg: Springer, 2016.

LEVY, D. L. Political contestation in global production networks. Academy of Management Review, 2008, 33 (4): 943-963.

LIVERMAN, D. Who governs, at what scale and at what price? Geography, environmental governance, and the commodification of Nature. Annals of the Association of American Geographers, 2004, 94 (4): 734-738.

MACLEOD, G. \& GOODWIN, M. Space, scale and state strategy: rethinking urban and regional governance. Progress in Human Geography, 1999, 23 (4): 503-527.

MAGGETTI, M. \& GILARDI, F. Network governance and the domestic adoption of soft rules. Journal of European Public Policy, 2014, 21 (9): 1293-1310.

MAYNTZ, R. Policy-Netzwerke und die Logik von Verhandlungssystemen. In: WINDHOFF-HÉRITIER, A. (editor) Policy-Analyse - Kritik und Neuorientierung. Politische Vierteljahresschrift (Sonderheft), Vol. 24. Opladen: Westdeutscher Verlag, 1993.

MAYNTZ, R. \& SCHARPF, F. (editores) Gesellschaftliche Selbstregelung und politische Steuerung. Frankfurt/ Main: Campus, 1995.

MONTERO, S. \& CHAPPLE, K. (editores). Fragile Governance and Local Economic Development: Theory and Evidence from Peripheral Regions in Latin America. New York: Routledge, 2019.

OSTROM, E. Coping with the tragedy of the commons. Annual Review of Political Science, 1999, 2 (1): 493.

PARK, S. H. \& UNGSON, G. R. Interfirm rivalry and managerial complexity: A conceptual framework of alliance failure. Organization Science, 2001, 12: 37-53.

PODOLNY, J. M. \& PAGE, K. L. Network forms of organization. Annual Review of Sociology, 1998, 24: 57-76.

POWELL, W. W. Neither market nor hierarchy: Network forms of organization. In: THOMPSON, G., FRANCES, J., LEVACIC, R. \& MITCHELL, J. (editores). Markets, Hierarchies and Networks. London: Sage, 1991, p. 265-276.

PROTA, L. Toward a Polanyian network analysis: market and non-market forms of coordination in the rice economy of Vietnam. Journal of Economic Geography, 2016, 16 (6): 1135-1160. 
PROVAN, K. G., FISH, A. \& SYDOW, J. Interorganizational networks at the network level: A review of the empirical literature on whole networks. Journal of Management, 2007, 33 (3): 479-516.

PROVAN, K. G. \& KENIS, P. Modes of network governance: Structure, management, and effectiveness. Journal of Public Administration Research \& Theory, 2008, 18: 229-252.

PROVAN, K. G. \& MILWARD, H. B. A preliminary theory of interorganizational network effectiveness: A comparative study of four community mental health systems. Administrative Science Quarterly, 1995, 40 (1): 1-33.

RHODES, R. A. W. Understanding governance: Ten years on. Organization Studies, 2007, 28 (8): 1243-1264.

RING, P. S. \& VAN DE VEN, A. H. Developmental processes of cooperative interorganizational relationships. Academy of Management Review, 1994, 19: 90-118.

ROWLEY, T., BEHRENS, D. \& KRACKHARDT, D. Redundant governance structures: An analysis of relational and structural embeddedness in the steel and semiconductor industries. Strategic Management Journal, 2000, 21: 369-386.

SÁNCHEZ HERNÁNDEZ, J. L. (editor) Espacios y Prácticas Económicas Alternativas en las Ciudades Españolas. Cizur Menor, Navarra: Thomson Reuters Aranzadi, 2019.

SCHÄFER, A. Netzwerk-Verfassungen: Institutionelle Lösungsansätze zur Überwindung kollektiver Handlungsprobleme in Verbundnetzwerken. Dissertation. Oldenburg: Universität Oldenburg. 2009

SCHARPF, F. Interaktionsformen: Akteurzentrierter Institutionalismus in der Politikforschung. Wiesbaden: VS Verlag, 2006.

SCOTT, M. Re-theorizing social network analysis and environmental governance:Insights from human geography. Progress in Human Geography, 2015, 39 (4): 449-463.

SEYFANG, G. Sustainable consumption, the new economics and community currencies: Developing new institutions for environmental governance. Regional Studies, 2006, 40: 781-791.

STABER, U. Steuerung von Unternehmensnetzwerken -Organisationstheoretische Perspektiven und soziale Mechanismen. In: SYDOW, J. \& WINDELER, A. (editores). Steuerung von Netzwerken Konzepte und Praktiken. Wiesbaden: Westdeutscher Verlag, 2000, p. 58-87.

SYDOW, J. (editor) Steuerung von Netzwerken: Konzepte und Praktiken. Opladen, Wiesbaden: Westdeutscher Verlag, 2000.

SYDOW, J. Strategische Netzwerke: Evolution und Organisation. Wiesbaden: Gabler, 2002.

SYDOW, J., SCHÜßLER, E. \& MÜLLER-SEITZ, G. (editores) Managing Interorganizational Relations: Debates and Cases London: Palgrave-Macmillan, 2016. 
TER WAL, A. L. J. \& BOSCHMA, R. A. Applying social network analysis in economic geography: framing some key analytic issues. The Annals of Regional Science, 2009, 43 (3): 739-756.

WEBER, M. Economy and Society, vol 1. Berkeley, CA: University of California Press, 1978.

WILLIAMSON, O. E. The Economic Institutions of Capitalism. Firms, Markets, Relational Contracting. New York: Free Press, 1985.

WILLIAMSON, O. E. The economics of governance. American Economic Review, 2005, 95 (2): 1-18.

WINDELER, A. Unternehmungsnetzwerke: Konstitution und Strukturation. Organisation und Gesellschaft. Wiesbaden: Westdeutscher Verlag, 2001.

YEUNG, H. W.-C. \& COE, N. M. Toward a dynamic theory of global production networks. Economic Geography, 2015, 91 (1): 29-58. 
